Portland State University

PDXScholar

6-16-2021

\title{
The Role of Cognitive Load and Individual Differences When Interpreting Human-Resource Data Visualizations
}

Zachary Hesson

Portland State University

Follow this and additional works at: https://pdxscholar.library.pdx.edu/honorstheses

Part of the Cognitive Psychology Commons, Human Resources Management Commons, Industrial and Organizational Psychology Commons, and the Personality and Social Contexts Commons Let us know how access to this document benefits you.

\section{Recommended Citation}

Hesson, Zachary, "The Role of Cognitive Load and Individual Differences When Interpreting HumanResource Data Visualizations" (2021). University Honors Theses. Paper 1098.

https://doi.org/10.15760/honors.1125

This Thesis is brought to you for free and open access. It has been accepted for inclusion in University Honors Theses by an authorized administrator of PDXScholar. Please contact us if we can make this document more accessible: pdxscholar@pdx.edu. 
Running head: COGNITIVE LOAD AND DATA VISUALIZATIONS

The Role of Cognitive Load and Individual Differences When Interpreting Human-Resource Data Visualizations

by

Zachary Hesson

An undergraduate honors thesis submitted in partial fulfillment of the requirements for the degree of

Bachelor of Science

in

University Honors

and

Psychology

Thesis Adviser

Dr. David E. Caughlin

Portland State University 


\begin{abstract}
Data visualizations (e.g., bar graph, dashboard) can be used as decision-support and storytelling tools that aid users' interpretation of sometimes complex information, including within the human resource management (HRM) context. As HRM evolves towards implementing more data-informed decisions, it is important to understand how users interpret data visualizations. The aims of this thesis are to (a) identify whether cognitive load affects the amount of time users spend arriving forming and interpretation and the accuracy of their interpretations, and (b) to evaluate whether cognitive load moderates the association between individual-difference variables and interpretation time and accuracy. The individual differences that are of particular interest are locus of control and the personality dimensions of extraversion, neuroticism, openness to experience, and conscientiousness. A sample of 58 undergraduate business students were randomly assigned to three different cognitive load levels (control, moderate, high), and each participant - irrespective of their group - responded to the same four data-visualization vignettes. Hypotheses were tested using a moderated multiple linear regression model. None of the proposed hypotheses were supported in the initial analysis, although after further analyses, cognitive load was a strong moderator of the association between neuroticism and interpretation accuracy for participants who experienced a moderate level of cognitive load, such that the association was negative when cognitive load was moderate. Theoretical and practical implications are included for developers of these data visualizations to keep in mind.
\end{abstract}

Keywords: data visualization, individual differences, five-factor model, locus of control, decision-support tool, cognitive load, human resource management. 
Today, data visualizations (e.g., bar graphs, scatter plots, line graphs) are used as decision-support and storytelling tools across different functional areas of organizations, including human resource management (HRM). Data visualizations can inform individuals who are not quantitative specialists on complex relationships between variables, which increases the accessibility of information within an organization (Sinar, 2015). And recent advances in computational power and informational technology have made data visualizations more readily available and easier to produce, which has led to a proliferation of use and an ever-expanding number of increasingly complex and sophisticated data-visualization display types. Despite this proliferation, researchers found that only $16 \%$ of organizations were using visualization tools effectively (DDI, The Conference Board \& EY, 2018). By failing to consider how datavisualization tools are deployed by organizations and interpreted by users (e.g., managers), users may be more likely to misinterpret the information displayed in a data visualization, potentially leading to poorer decision making.

When considering the HRM context specifically, improving how data visualizations are designed and deployed might allow HR managers, or other consumers of HR data, to make better decisions about human capital. In terms of the design, one consideration is the use of chartjunk within a data-visualization display. Chartjunk refers to unnecessary visual elements that are placed onto a display that do not facilitate interpretation of the data being presented given the task at hand (Tufte, 1983). Whether chartjunk aids or hinders the interpretability of data visualizations is still an ongoing debate (Bailey \& Pregill, 2014). In terms of the deployment of data visualizations, extraneous workplace cognitive demands (e.g., noise, distractions, multitasking, role overload) may also impair users' task performance as they attempt to interpret key information displayed within a data visualization. 
The tenets of cognitive load theory (CLT) offer a way to understand how extraneous workplace cognitive demands may affect how users process and interpret information displayed in a data visualization (Sweller, 2010). Specifically, CLT posits that individuals who experience higher cognitive loads are more likely to experience distractor interference. This theory expands upon the idea that individuals have a limited working memory capacity, and when the working memory load is too high, it has the potential to attenuate their ability to process information effectively. Lavie (2010) mentions that "Cognitive control functions are loaded when people have to switch back and forth between different tasks or when people have to actively maintain in working memory some task unrelated material during task performance" (p. 147). Within a busy workplace environment, it is likely that multiple concurrent tasks and other types of cognitive demands that add to working memory demands, leading to higher overall cognitive load.

Limiting the amount of extraneous cognitive load that individuals experience is one of the primary focuses of CLT research (Sweller, 1994), as decreasing the amount of cognitive load that an individual experiences could lead to overall better task performance given that more working memory resources are available to attend to the task at hand. Given that, this study aims to investigate the association between extraneous cognitive load and data-visualization interpretation efficiency and effectiveness.

Cognitive load, however, is not the only factor that may affect how users interpret data visualizations. Namely, users' levels of certain individual-difference variables may affect how users interpret data visualizations, and may affect how experienced cognitive impacts such interpretations. The focal individual-difference variables that this thesis focuses on are extraversion, neuroticism, openness to experience, conscientiousness, and locus of control, 
which prior research has found to have implications for job performance (Barrick \& Mount 1991; Green \& Fisher, 2010). Additionally, to my knowledge there is no research on how individual differences and cognitive load interact with each other when examining the interpretation of data visualizations. In my thesis, I aim to investigate whether experienced cognitive load moderates the association between individual differences and data-visualization interpretation efficiency and effectiveness.

My thesis makes three primary contributions. First, this study has the potential to contribute to the understanding of how users' experience of extraneous sources of cognitive load may affect the speed and accuracy of how information is interpreted when data visualizations are used as decision-support tools. In this way, my aim is to contribute to the CLT literature by applying theoretical tenets to the HR data visualization context. Second, this research will contribute to the small but growing body of research around individual differences and the interpretation of data visualizations, as well as attempt to identify a previously unexplored interaction effect between individual differences and cognitive load in relation to datavisualization interpretation efficiency and effectiveness.

In the sections that follow in the introduction, I begin by reviewing the research around data visualizations and how it applies to a HRM setting, followed by an examination how cognitive load may affect how users interpret data-visualizations as sources of information for completing tasks. After that, I review what is currently known about individual differences and task performance in general, and how cognitive load may moderate the association between individual-difference variables and data-visualization interpretation efficiency and effectiveness. 


\section{Data Visualizations}

A data visualization can be defined as "a set of processes via which data are graphically displayed and interpreted with a particular goal in mind to ultimately derive meaning in the form of information and knowledge" (Caughlin \& Bauer, 2019, p. 95). A number of other closerelated terms are often used interchangeably when referring to a data visualization, such as a plot, chart, graph, diagram, or graphical display. Classic examples of data visualizations include the bar graph, line graph, and table. Data visualizations can display information about a single variable or the associations between two more variables.

\section{Data Visualizations as Storytelling and Decision-Support Tools}

Data visualizations can be conceptualized as both storytelling and decision-support tools. First, as a storytelling tool, data visualizations can be designed and deployed to report facts and convey narratives, which can improve communication and more generally aid interpretation. In general, storytelling can make complex information more accessible and more memorable (Roels et al., 2017). Given that a data visualization is a type of storytelling tool, it stands to reason then that a thoughtfully designed and deployed data visualization has the potential to make information more accessible and memorable to users in a variety of settings, including in organizations. Second, data visualizations can also be deployed as decision-support tools. The information displayed within a data visualization can be interpreted with the goal of finding solutions to problems, finding answers to questions, and building knowledge on a particular topic, all of which may pave the way for more efficient and effective interpretations and higher overall task performance. 


\section{Role of Data Visualizations in Human Resource Management}

In today's organizations, managers frequently rely on data visualizations to facilitate task completion and decisions relating to staffing, products, and other essential operations. Among HR managers, specifically, data visualizations are commonly used to describe, explain, and understand the transactions and activities of the workforce. For example, a simple bar graph might be used by a team of training specialists to show whether employees who participated in a new version of a training program had higher average post-assessment scores than those who participated in an older version of a training program. As another example, a line graph might be used for workforce planning purposes to illustrate how headcount has changed over time by specific organizational units (e.g., marketing, research and development, and operations unit). Finally, a variety of data visualization display types - including data visualizations arranged in a dashboard - may be used to summarize and describe employee engagement survey responses.

\section{Design and Deployment of Data Visualizations}

When determining how to best design and deploy a data visualization, it is important to consider the characteristics of the intended user and their experiences, the message intended to be communicated using the displayed information, and the tasks users may complete using the displayed information. These considerations have implications for how efficiently and effectively the intended user interprets the information conveyed in the data visualization and ultimately how well they perform associated work tasks. To that end, Caughlin and Bauer (2019) proposed an integrated framework that highlighted some important considerations when designing and deploying data visualizations. Specifically, the integrated framework suggested that display characteristics (e.g., display type, display format), user characteristics (e.g., individual differences, experience, familiarity), and the tasks and objectives the user may 
accomplish using the data visualization (e.g., compare data values, identify change over time) have implications for the user's interpretation performance in terms of efficiency and effectiveness. Following a portion of this framework, this thesis focuses on the association between user characteristics and interpretation efficiency (e.g., speed) and effectiveness (e.g., accuracy). Such user characteristics could include the different working memory demands that the users are concurrently exposed to (e.g., distractions in the work environment, multitasking) as well as relatively stable dispositional characteristics like individual differences.

If users misinterpret or misidentify information that is conveyed in a data visualization, then it is likely that overall interpretation task performance will be affected negatively. For example, display characteristics (e.g., trends, display format) can greatly influence how a user interprets information contained in a data visualization (Reb \& Cropanzano, 2007). Further, different user characteristics, such as dispositional tendencies and concurrent work cognitive demands, might lead to heterogeneity in interpretations among a group of users. Thus, in addition to the design of a data visualization display, it is important to consider how the data visualization is deployed and to whom, which implies the importance of being aware of and/or sensitive to how end users with different characteristics and experiences might interpret a data visualization differently. After all, if a user does not interpret the intended message of the data visualization in an efficient or effective manner, then that user may perform poorly when applying their interpretation in the context of a work task.

\section{Data-Visualization Interpretation and Task Performance}

When available, HR managers may interpret information presented in data visualizations to aid completion of tasks, which means that data-visualization interpretation has potential implications for task performance. Broadly speaking, task performance can be conceptualized as 
one dimension of job performance, where other dimensions include organizational citizenship behavior and counterproductive work behavior (Rotundo \& Sackett, 2002). Task performance can be defined as the "behaviors that contribute to the production of a good or the provision of a service" (Rotundo \& Sackett, 2002, p. 67), which are not limited to the behaviors that appear in the job description. Consistent with Caughlin and Bauer (2019), a well-designed and -deployed data visualization may have the potential to improve interpretation efficiency and effectiveness and ultimately enhance task performance.

\section{Interpretation Efficiency: Time}

In terms of interpretation efficiency, a single data visualization can illustrate graphically a difference or association that would otherwise require a lengthy text explanation, thereby reducing the amount of time that a busy manager or professional needs to spend when interpreting relevant information for the purposes of performing a work task. For example, imagine an HR manager who is tasked with identifying which sales employees might be in need of performance development plans. The manager could use a line graph like the one presented in Figure 5 to efficiently identify which sales employees have had consistently declining performance in recent years and thus might be in need of a performance development plan. By comparison, a text description of the same information presented visually in the line graph could be lengthy and thus lead the manager to spend more time arriving at a decision regarding who needs a performance development plan.

It is important to note that in this context, greater interpretation efficiency corresponds to a lower elapsed time and to a faster speed. To avoid confusion and to make subsequent hypotheses more intuitive, decision-making efficiency will from this point forward be referred to as interpretation time or time spent. 


\section{Interpretation Effectiveness: Accuracy}

In terms of decision-making effectiveness, a data visualization can be used to summarize sometimes complex information (e.g., differences, associations, trends). Extending the same example from the previous paragraph, a line graph depicting performance trends over recent years for a group of employees may be less cognitively demanding and more digestible than a densely written account of the same information. Accordingly, a well-designed and -deployed line graph, for example, may make help the HR manager more accurately interpret and evaluate the performance-trend data, thereby facilitating their ability to perform their managerial tasks.

\section{Cognitive Load Theory}

Cognitive load theory (CLT; Sweller, 2010) posits that there are three different types of cognitive load: (a) intrinsic cognitive load (i.e., innate difficulty of a task), (b) extraneous cognitive load (i.e., how information is presented), and (c) germane cognitive load (i.e., working memory resources that convert into schemas which result in long term memory). Regardless of the type, cognitive load consists of two distinguishable components: mental load and mental effort (Paas, 1992). Mental load is the amount of load stemming from the task and how it is presented, whereas mental effort refers to how many mental resources are being spent on that specific task at hand.

CLT posits that individuals who experience greater cognitive load are more likely to experience distractor interference (Murphy et al., 2016). This stems from the idea that individuals have limited working memory, and when working memory demands are high, the capacity to process information becomes strained. Extraneous cognitive load and intrinsic cognitive load are additive, meaning that if you have higher levels of one, then you must strive for lower levels of the other (van Merriënboer \& Sweller, 2005). If both intrinsic and extrinsic 
levels of cognitive load are too high, then learning will be impaired and individuals will likely not grasp the information (Sweller, 1994).

To operationalize cognitive load, a separate working memory task will be given to certain participants. This task will be similar to the one found in Lavie (2010) and will help to better simulate the complexities of the workplace. Given that cognitive load adds to the amount of mental load and mental effort being spent interpreting a data visualization as part of a work task, it stands to reason that individuals who are exposed to higher levels of cognitive load will need to spend more time interpreting the information contained in a data visualization and that higher levels of cognitive load will lead to more interpretation errors - or in other words lower accuracy.

Hypothesis 1: Cognitive load is positively associated with data-visualization interpretation time.

Hypothesis 2: Cognitive load is negatively associated with data-visualization interpretation accuracy.

\section{Individual Differences \& Task Performance}

Individual differences in personality are unique variations within certain characteristics that differentiate individuals from one another, where personality can be defined as referring to those characteristics of the person that account for consistent patterns of feeling, thinking, and behaving (Pervin et al., 2005, p.6). They are an integral part of user characteristics (Caughlin \& Bauer, 2019), and as such, they are a necessary point of consideration for data-visualization interpretation efficiency (i.e., time spent) and effectiveness (i.e., accuracy) as it relates to task performance. As shown in the conceptual framework depicted in Figure 1, I will investigate 
whether cognitive load moderates the association between different individual-difference variables and data-visualization interpretation efficiency and effectiveness.

\section{Figure 1}

Conceptual framework for present study

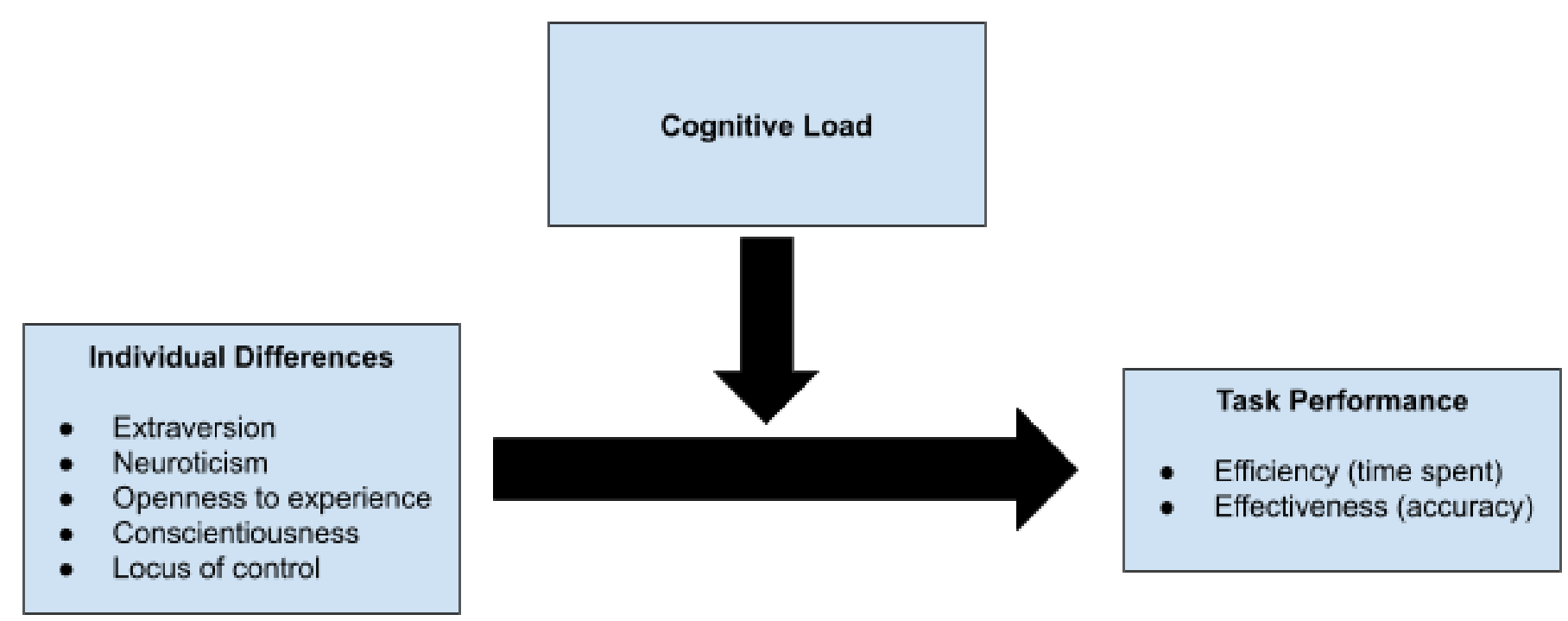

\section{Locus of Control}

Locus of control (LOC) has been chosen as a main area of focus considering the impact that it has on a variety of outcomes, such as academic and job performance (Green \& Fisher, 2010). Conventionally, LOC is operationalized such that those with higher scores have a higher internal LOC and those with lower scores have a higher external LOC (e.g., Spector, 1988). Further, those with a higher LOC look to themselves for direction, whereas those with a lower LOC rely more on outside elements, such as a supervisor or manager (Spector, 1982).

Because individuals with a higher LOC tend to demonstrate better problem-solving skills and thus are more likely to persist when a task gets more challenging (Weiss \& Sherman, 1973), I argue that those who have a higher LOC and who experience higher cognitive load will spend 
more time interpreting a data visualization than those who experience a lower cognitive load. In other words, I predict that cognitive load moderates the association between LOC and interpretation time, such that the association is more positive when cognitive load is higher.

Hypothesis 3: Cognitive load moderates the association between LOC and datavisualization interpretation time, such that under conditions of higher cognitive load the association between LOC and interpretation time will be more positive.

Given that those with a higher LOC tend to demonstrate better problem-solving skills (Weiss \& Sherman, 1973), one would expect the association between LOC and datavisualization interpretation accuracy to be positive, such that those with a higher LOC arrive at more accurate interpretations. Further, because cognitive load is associated with greater mental effort and mental load, it stands to reason that the positive association between LOC and interpretation accuracy will be attenuated under conditions of higher cognitive load.

Hypothesis 4: Cognitive load moderates the association between LOC and datavisualization interpretation accuracy, such that under conditions of higher cognitive load the association between LOC and interpretation accuracy will be less positive.

\section{Five-Factor Model}

Researchers have been interested in the relationship between the five-factor model (FFM) and task performance for decades, and the measures of the FFM (i.e., Big Five) are regularly used in personnel selection. The FFM consists of five personality dimensions: extraversion, openness to experience, neuroticism, agreeableness, and conscientiousness. Meta-analytic evidence has shown that these five dimensions tend to show small associations with overall job performance, with conscientiousness showing the largest association with overall job 
performance across different occupation types (Barrick \& Mount, 1991). Further, in that same meta-analytic investigation, when focusing on the manager occupational group, these associations were of the same magnitude or slightly larger, which is particularly relevant to this study given my focus on HR managers as the target population. Interestingly, a subsequent metaanalytic investigation by $\mathrm{Oh}$ and colleagues (2011) found that the magnitude of associations between the five personality dimensions and overall job performance tend to be larger in magnitude when personality is rated by observers as opposed to self-rated. With respect to how the FFM might influence individuals' data-visualization interpretations, relatively little research has been published in this area to date. To that end, Liu and colleagues (2020) reviewed the datavisualization literature and found that only a few studies that looked at the potential impact of personality on how individuals' interpret the information displayed in data visualizations, leading the authors to argue that we need more research in this area. Heeding this call, this study aims to address this topic. Next, I describe each FFM dimension and how it might affect datavisualization interpretation.

Neuroticism. Neuroticism is defined as the propensity to experience negative emotions such as stress, nervousness, or moodiness (Goldberg, 1992). Neuroticism is sometimes referred to by the opposite end of the same continuum: emotional stability. In general, neuroticism tends to be negatively associated overall job performance (Barrick \& Mount, 1991). Further, those with higher levels of neuroticism have been found to interpret data visualizations and complete associated tasks in less time but with fewer insights reported (Green \& Fisher, 2012). Given this, I predict that neuroticism will be negatively associated with data-visualization interpretation time. In the presence of higher cognitive load, I argue that the negative association will be attenuated. 
Hypothesis 5: Cognitive load moderates the association between neuroticism and datavisualization interpretation time, such that under conditions of higher cognitive load the association between neuroticism and interpretation time will be less negative.

Based on Green and Fisher's (2012) that those with higher neuroticism tend to glean fewer insights from data visualizations, I expect a negative association between neuroticism and data-visualization interpretation accuracy. Moreover, I predict that this association will become more negative when individuals are faced with greater mental effort and mental load that is associated with higher cognitive load.

Hypothesis 6: Cognitive load moderates the association between neuroticism and datavisualization interpretation accuracy, such that under conditions of higher cognitive load the association between neuroticism and interpretation accuracy will be more negative.

Extraversion. Extraversion can be briefly described as how likely individuals are to interact with the outside world (Goldberg, 1992). The construct is typically conceptualized as existing along a introversion-extraversion continuum, where individuals who have higher extraversion tend to be more outgoing, and individuals who have lower extraversion tend to be less outgoing and less likely to engage with others. Similar to their aforementioned findings regarding neuroticism, Green and Fisher (2012) found that those with higher extraversion tended to interpret data visualizations and complete associated tasks in less time but with fewer insights reported. Given that, I expect cognitive load to moderate the associations between extraversion and data-visualization interpretation time and accuracy in the same manner as described above for neuroticism. 
Hypothesis 7: Cognitive load moderates the association between extraversion and datavisualization interpretation time, such that under conditions of higher cognitive load the association between extraversion and interpretation time will be less negative.

Hypothesis 8: Cognitive load moderates the association between extraversion and datavisualization interpretation accuracy, such that under conditions of higher cognitive load the association between extraversion and interpretation accuracy will be more negative.

Openness to experience. Openness to experience can be described as being imaginative, creative, and curious about the world (Goldberg, 1992), and sometimes this dimension is referred to as intellect. Individuals that scored high on this personality dimension tend to have better problem-solving skills (Myszkowski et al., 2015). Ziemkiewicz and Kosara (2009) found that those that were higher on openness were more comfortable when working with conflicting visual and verbal metaphors and they also add that users that are high on this dimension were likely to understand the visual metaphor based on their spatial ability. This suggests that openness to experience may be an important personality dimension in the context of data-visualization interpretation, and specifically I expect that those with higher openness to experience will spend less time interpreting data visualizations and will make more accurate interpretations. With respect to cognitive load, I predict that both associations will be attenuated under conditions of higher cognitive load due to the associated greater mental demands.

Hypothesis 9: Cognitive load moderates the association between openness to experience and data-visualization interpretation time, such that under conditions of higher 
cognitive load the association between openness to experience and interpretation time will be less negative.

Hypothesis 10: Cognitive load moderates the association between openness to experience and data-visualization interpretation accuracy, such that under conditions of higher cognitive load the association between openness to experience and interpretation accuracy will be less positive.

Conscientiousness. Conscientiousness can be described as the propensity to remain organized, reliable, and thorough (Goldberg, 1992), and people who are high in conscientiousness tend to be task-oriented and dutiful. As mentioned above, this dimension of the FFM is the most consistent predictor of job performance across all job types (Barrick \& Mount, 1991). Yet, to date, research has not found conscientiousness to be associated with task performance involving data-visualization interpretation (Brown et al., 2014; Ziemkiewicz \& Kosara, 2009), which may be an artifact of the types of visualizations used in these studies. With that being said, given that people who are higher in conscientiousness tend to display more selfdiscipline and tend to be more task oriented, I suspect that those with higher conscientiousness tend to spend more time interpreting data visualizations and tend to make more accurate interpretations. I predict that the expected positive association between conscientiousness and data-visualization interpretation time will be augmented under conditions of higher cognitive load due to the associated mental effort and load, and conversely I predict that the expected positive association between conscientiousness and data-visualization interpretation accuracy will be attenuated under conditions of higher cognitive load.

Hypothesis 11: Cognitive load moderates the association between conscientiousness and data-visualization interpretation time, such that under conditions of higher 
cognitive load the association between conscientiousness and interpretation time will be more positive.

Hypothesis 12: Cognitive load moderates the association between conscientiousness and data-visualization interpretation accuracy, such that under conditions of higher cognitive load the association between conscientiousness and interpretation accuracy will be less positive.

Agreeableness. Agreeableness can be defined as the proclivity to be kind, trusting, or affable amongst a group of individuals (Goldberg, 1992). Although some researchers have investigated whether agreeableness is associated with task performance (Brown et al., 2014; Ziemkiewicz \& Kosara, 2009), the results have been inconclusive. Given the construct's focus on harmony with others, it is unlikely that this dimension of personality will be associated with data-visualization interpretation in any systematic manner; thus, I have no hypotheses involving agreeableness.

\section{Method}

\section{Design}

A between-subjects design was used in this study, where the between-subjects factor consisted of three cognitive-load levels (control, moderate, high). Specifically, all participants completed individual-difference and demographic measures and were subsequently randomly assigned to one of the three cognitive-load conditions. After assigned to a condition, each participant was then exposed to four data-visualization tasks, which varied in terms of their chart embellishments and chartjunk. After reviewing each data visualization, participants were then measured on the time and accuracy with which they completed each data-visualization task and their confidence level regarding the accuracy of their decision. 


\section{Sample and Participants}

Undergraduate business students were recruited for this study using a large university's online participant recruitment platform called the SONA system. Business students were chosen given that these students should be familiar with data visualizations and management principles, potentially limiting the number of potential confounding variables associated with these tasks. Eighty-one students were recruited using this platform; however, 16 students were removed for failing an attention check item, and 7 students were removed for completing the entire survey in less than 5 minutes - the latter of which was an a priori threshold set to exclude participants who completed the study too hastily. Of the 58 participants who were retained, $31.0 \%(n=18)$ were in the control group, $32.8 \%(n=19)$ were in the moderate cognitive load group, and $36.2 \%(n=$ 21) were in the high cognitive load group. In regard to their gender identities, $24.1 \%(n=14)$ identified as men, $74.1 \%(n=43)$ identified as women, and $1.7 \%(n=1)$ identified as genderqueer. The race and ethnicity statistics are as follows: $37.9 \%(n=22)$ identified as White, 17.2\% $(n=10)$ identified as Hispanic or Latino, $22.4 \%(n=13)$ indicated that they were Asian, 3.4\% $(n=2)$ identified as American Indian or Alaska Native, 3.4\% $(n=2)$ identified as Black or African American, and 15.5\% $(n=9)$ identified with two or more races or ethnicities. Participants' ages ranged from 18 to 41 years, with an average age of 24.7 years and a standard deviation of 5.3 .

\section{Measures}

The individual-differences measures were administered at the beginning of the study, and the data-visualization interpretation efficiency and effectiveness measures were administered after each data-visualization task. 


\section{Individual Differences}

Participants completed measures intended to assess the Five-Factor Model (FFM) and locus of control (LOC). Sixteen items from the Mini-IPIP (Donnellan et al., 2006; Goldberg, 1992; Goldberg 1999) were used to assess the following 4-item dimensions: extraversion, openness to experience, neuroticism, and conscientiousness; agreeableness items were not used from this measure because I did not make any hypotheses associated with that personality dimension. These dimensions were assessed using a 5-point Likert agreement response format, ranging from 1 ("strongly disagree") to 5 ("strongly agree"). Each dimension had 4 items for its respective subscale, with 2 reverse-coded items included in the extraversion, neuroticism, and conscientiousness dimensions and 3 reverse-coded items included in the openness to experience dimension. Sample items include: extraversion ("I am the life of the party."; "I don’t talk a lot. (reverse-coded)"; $\alpha=.64$ ), openness to experience ("I have a vivid imagination."; "I am not interested in abstract ideas. (reverse-coded)"; $\alpha=.70$ ), neuroticism ("I have frequent mood swings."; "I am relaxed most of the time. (reverse-coded)"; $\alpha=.61$ ), and conscientiousness ("I get chores done right away."; "I make a mess of things. (reverse-coded)"; $\alpha=.45$ ). Individuals that were higher on these respective scales exhibited stronger characteristics of the construct.

LOC was measured using a 7-item scale (Spector, 1988; $\alpha=.74$ ). This scale used a 5point Likert response format, ranging from 1 ("strongly disagree") to 5 ("strongly agree"). There were 6 items on this scale that were scored regularly, and 1 item was reverse-coded. The reversecoded item was dropped to increase internal consistency reliability, leaving 6 items. Sample items for LOC included: "A job is what you make of it." and "If you know what you want out of a job, you can find one that gives it to you.”. Higher scores on this scale are associated with higher internal LOC, whereas lower scores are associated with a higher external LOC. 


\section{Data-Visualization Interpretation Efficiency and Effectiveness Measures}

Data-visualization interpretation efficiency and effectiveness were operationalized as time and accuracy, respectively. Time was assessed by determining how long it took a participant to respond to each question after interpreting each data visualization task. A composite variable was created for participants by taking the average response time for all of the participants successfully completed tasks. Participants who were unable to complete the task within 60 seconds had their responses removed for that question, as the instructions were to select a response quickly.

Accuracy was assessed based on whether a participant interpreted a data visualization correctly and thus answered the associated task question correctly (i.e., decision-making accuracies). Correct responses were given a value of 1 and incorrect responses were given a value of 0 . Composite variables were created for each participant by using the sum of all the correct responses, with possible scores ranging from 0 to 4 . Out of the 58 participants who completed the survey, $15.52 \%(n=9)$ answered 0 correctly, $29.31 \%(n=17)$ answered 1 correctly, $31.03 \%(n=18)$ answered 2 correctly, $22.41 \%(n=13)$ answered 3 correctly, and $1.7 \%(n=1)$ answered all 4 questions correctly.

As an exploratory data-visualization interpretation measure, I created a confidenceaccuracy measure. to determine an individual's confidence level in the accuracy of their response to each question, participants self-reported how confident they were with each response ranging from 0 ("not at all confident") to 100 ("very confident"). These values were then multiplied by 1 or -1 depending on whether the participant responded correctly (1) or incorrectly (-1), and an overall confidence-accuracy estimate was computed based on the average confidence-accuracy for each of the four data-visualization tasks. 


\section{Procedure and Materials}

Participants were recruited through a large university's business school using the SONA platform, and students received extra credit for participating courses for participating in a certain number of research studies. They were able to log into the survey remotely at their own leisure. First, they clicked on the Qualtrics survey and completed the informed consent page. Next, they completed the individual difference measures. Afterwards, they were randomly placed into one of the three cognitive load conditions (control, moderate, high). Participants who were assigned to the moderate and high conditions were given a working memory task that they were to complete in addition to the primary data-visualization task. This task was completed after the data-visualization task and changed after each task was completed. Every participant was then given a task where they were prompted to answer a specific question related to the data visualization, and this was completed a total of four times with a different task and data visualization each time. Participants' interpretation time and accuracy were recorded after each data-visualization task was completed.

\section{Cognitive-Load Manipulation}

To ensure that cognitive load is being operationalized as opposed to perceptual load, participants were randomly assigned to one of three conditions. The first group did not receive a working-memory task and served as the control group. The second group received a moderatelevel working-memory task where they are to memorize a four-digit alphanumeric sequence prior to the main task (see Figure 2). A different sequence was repeated after each primary datavisualization task. The third group received high-level working-memory, which was similar to the moderate-level task except that the alphanumeric sequence had no predictable pattern (see Figure 3). These secondary working-memory tasks changed after each primary task was 
completed, so that there were four unique working-memory tasks that accompanied the four primary data-visualization tasks. Participants who were able to return at least $50 \%$ of the sequence in any order were considered to have successfully completed the working-memory task. Those who did not successfully complete the working-memory task had null values entered for their data-visualization interpretation measures.

\section{Figure 2}

Moderate cognitive-load condition

\section{R3S4 J4K5 D6E5 C8D9}

Note. The alphanumeric sequences in this moderate cognitive load condition have a relatively predictable pattern, making this sequence easier to recall.

\section{Figure 3}

High cognitive-load condition

\section{B9X1 L7K4 N3K6 P2F9}

Note. The alphanumeric sequences in this high cognitive-load condition do not have predictable patterns, as these sequences are random. These sequences are more difficult to remember than the moderate cognitive-load condition.

\section{Data-Visualization Interpretation Tasks}

Specific prompts were given to individuals after the working memory task as they viewed the data visualization. Participants were asked to complete specific spatial tasks while analyzing the four different data visualizations one by one. The data visualizations consisted of two bar 
graphs (Figure 4) and two line graphs (Figure 5). All of the data visualization tasks had eight possible responses. 


\section{Figure 4}

Bar graph data-visualization tasks

Annual Report

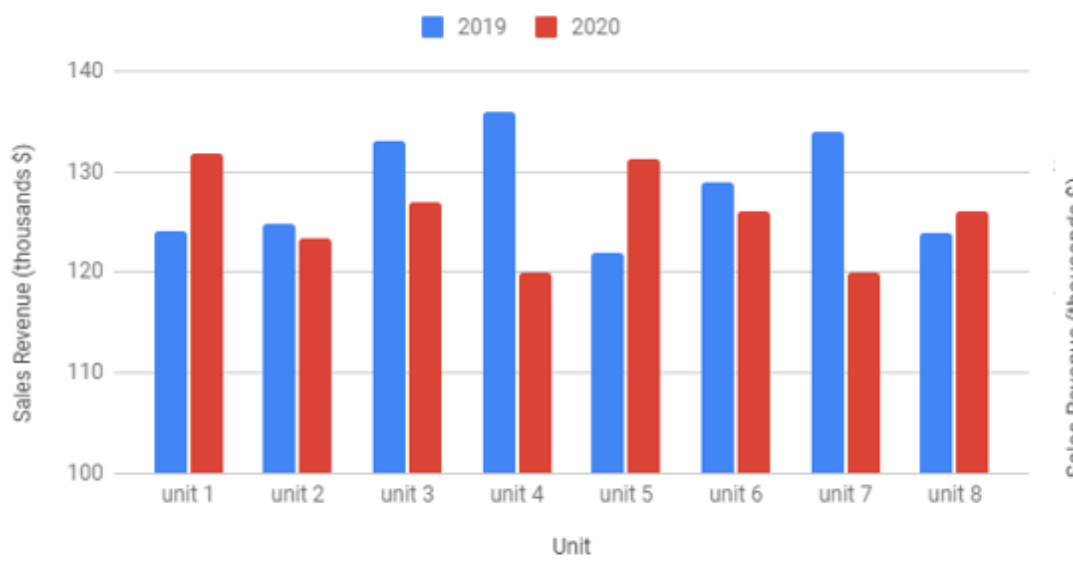

Which unit's combined sales revenue for 2019 and 2020 is the largest?

\section{Annual Report}

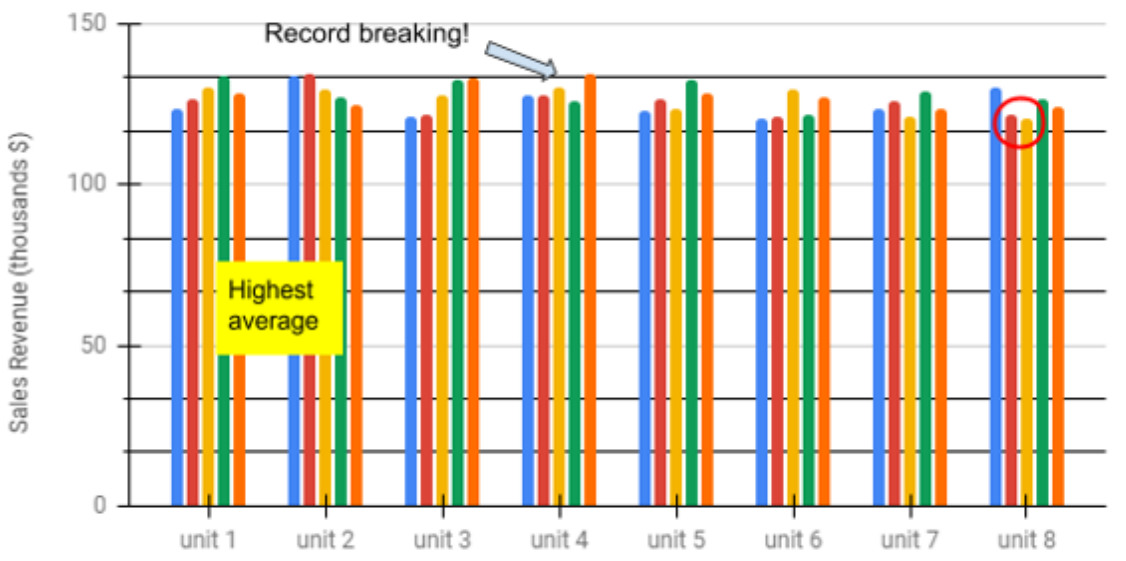

Which unit had the lowest number of sales revenue in 2019? 
Figure 5

Line graph data-visualization tasks

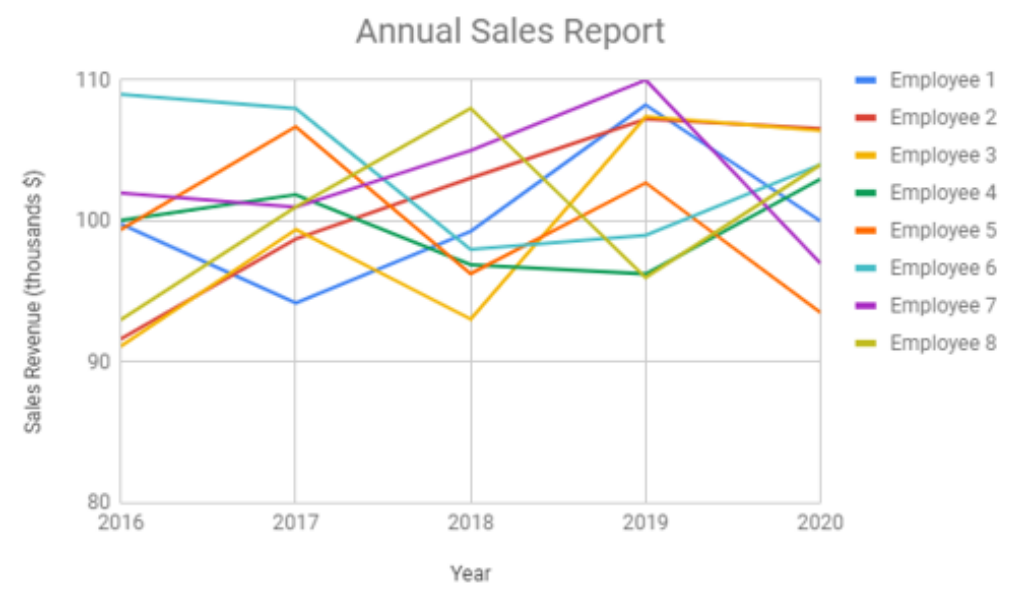

Between the years 2016 and 2020, which employee had the lowest amount of variability in their sales revenue?

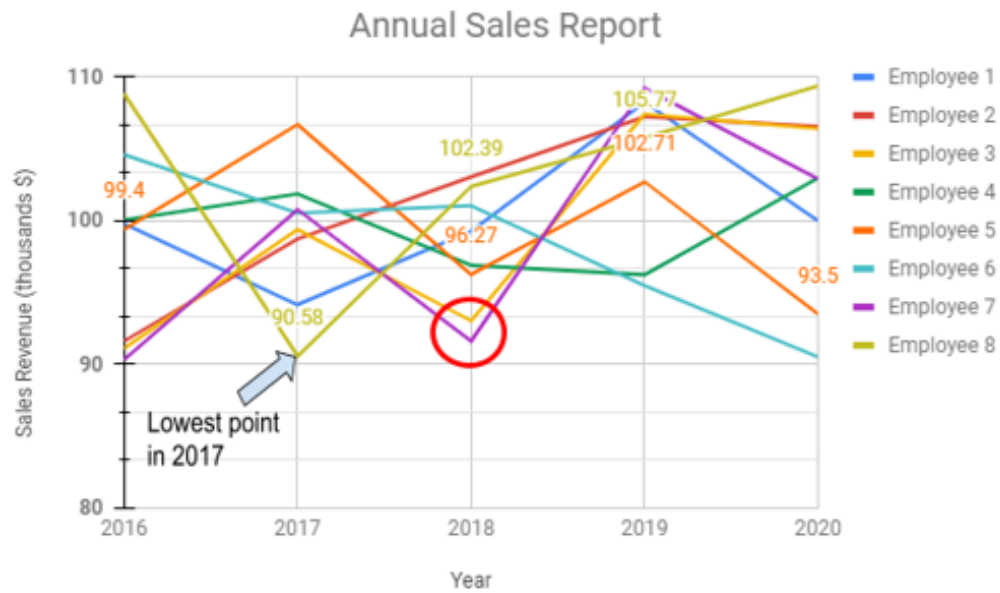

Between 2016 and 2020, which employee had the largest decrease in sales revenue? 
COGNITIVE LOAD AND DATA VISUALIZATIONS

\section{Results}

Table 1 shows the correlations between the focal non-manipulation variables. Because conscientiousness had poor levels of internal consistency reliability $(\alpha=.45)$, Hypotheses 11 and 12 were not tested. Extraversion and neuroticism had questionable levels of internal consistency reliability ( $\alpha=.64$ and $\alpha=.61$, respectively). Locus of control (LOC) and openness to experience had adequate levels of internal consistency reliability $(\alpha>.70)$. Table 2 includes the means and standard deviations for each of the focal variables for each of the three cognitive-load conditions. Tables 1, 3, and 4 were created using the apaTables R package (Stanley, 2021). 
COGNITIVE LOAD AND DATA VISUALIZATIONS

Table 1

Zero-order correlations with confidence intervals for focal non-manipulation variables

\begin{tabular}{|c|c|c|c|c|c|}
\hline Variable & 1 & 2 & 3 & 4 & 5 \\
\hline \multicolumn{6}{|l|}{ 1. Speed } \\
\hline 2. Accuracy & $\begin{array}{l}.31 * \\
{[.05, .52]}\end{array}$ & & & & \\
\hline 3. Locus of control & $\begin{array}{c}.01 \\
{[-.24, .27]}\end{array}$ & $\begin{array}{c}.04 \\
{[-.22, .30]}\end{array}$ & & & \\
\hline 4. Neuroticism & $\begin{array}{c}-.33 * \\
{[-.54,-.08]}\end{array}$ & $\begin{array}{c}-.33^{*} \\
{[-.54,-.07]}\end{array}$ & $\begin{array}{c}-.04 \\
{[-.29, .22]}\end{array}$ & & \\
\hline 5. Extraversion & $\begin{array}{c}-.01 \\
{[-.27, .25]}\end{array}$ & $\begin{array}{c}.11 \\
{[-.15, .36]}\end{array}$ & $\begin{array}{c}.01 \\
{[-.25, .27]}\end{array}$ & $\begin{array}{c}.05 \\
{[-.22, .30]}\end{array}$ & \\
\hline 6. Openness to experience & $\begin{array}{l}.24 \\
{[-.02, .47]}\end{array}$ & $\begin{array}{c}.01 \\
{[-.25, .26]}\end{array}$ & $\begin{array}{c}-.06 \\
{[-.31, .20]}\end{array}$ & $\begin{array}{c}-.05 \\
{[-.30, .21]}\end{array}$ & $\begin{array}{c}-.11 \\
{[-.36, .15]}\end{array}$ \\
\hline
\end{tabular}

Note. $n=58$. Values in square brackets indicate the $95 \%$ confidence interval for each correlation. The confidence interval is a plausible range of population correlations that could have caused the sample correlation (Cumming, 2014). * indicates $p<.05$. ** indicates $p<.01$. 
COGNITIVE LOAD AND DATA VISUALIZATIONS

Table 2

Means and standard deviations of focal variables by levels of cognitive load

(control, moderate, high) and across cognitive load levels (overall)

\begin{tabular}{lrrrrrrrr}
\hline & \multicolumn{2}{c}{ Control } & \multicolumn{2}{c}{ Moderate } & \multicolumn{2}{c}{ High } & \multicolumn{2}{c}{ Overall } \\
\cline { 2 - 9 } \multicolumn{1}{c}{ Variables } & \multicolumn{1}{c}{$M$} & \multicolumn{1}{c}{$S D$} & \multicolumn{1}{c}{$M$} & \multicolumn{1}{c}{$S D$} & \multicolumn{1}{c}{$M$} & \multicolumn{1}{c}{$\boldsymbol{M}$} & \multicolumn{1}{c}{$S D$} \\
\hline Speed & 30.43 & 8.88 & 31.67 & 10.28 & 32.29 & 8.64 & 31.51 & 9.15 \\
Accuracy & 2.00 & 1.14 & 1.58 & 1.12 & 1.43 & .87 & 1.66 & 1.05 \\
Locus of & 3.92 & .72 & 3.75 & .76 & 3.71 & .63 & 3.79 & .70 \\
$\quad$ control & & & & & & & & \\
Neuroticism & 2.97 & .76 & 2.87 & .93 & 3.08 & .76 & 2.98 & .81 \\
Extraversion & 2.97 & 1.00 & 3.00 & .71 & 2.77 & .92 & 2.91 & .87 \\
Openness to & 3.56 & .93 & 3.5 & .76 & 3.81 & .69 & 3.63 & .79 \\
experience & & & & & & & & \\
\hline
\end{tabular}

Note. $M$ and $S D$ are used to represent mean and standard deviation, respectively.

\section{Hypotheses 1 and 2}

One-way ANOVAs were used to determine whether there were significant differences in data-visualization interpretation time and accuracy across the three different groups. For Hypothesis 1, I predicted that cognitive load would be positively associated with datavisualization interpretation time. Because there were no significant differences in time between the three cognitive-load groups $(F 2,55=.201, p=.81)$, Hypothesis 1 was not supported. For Hypothesis 2, I predicted that cognitive load would be negatively associated with datavisualization interpretation accuracy. Because there were no significant differences in the number of accurate interpretations between the three cognitive-load groups $(F 2,55=1.53, p=$ $0.23)$, Hypothesis 2 was not supported.

\section{Hypotheses 3-10}

Moderated multiple linear regression (MMLR) was used to test Hypotheses 3-10, as these hypotheses involved a continuous predictor variable (i.e., LOC, neuroticism, extraversion, openness to experience) and a categorical moderator variable (i.e., cognitive-load variable) with 
data-visualization interpretation time and accuracy as the outcome variables. [Please note that, as mentioned above, Hypotheses 11 and 12 were dropped due to the low internal consistency reliability of the conscientiousness measure.] Because the cognitive-load variable had three levels (control, moderate, high), two dummy variables were created with the control condition as the reference group. Each hypothesis was tested using hierarchical linear regression as follows: (a) a nested additive model was estimated in which one of the individual-difference variables and the two cognitive-load dummy variables were specified as predictors, and one of the datavisualization interpretation measures (i.e., time or accuracy) was specified as the outcome; (b) a full model was estimated which include the same variables as the nested model as well as the product terms between the individual-difference variable and the two dummy variables; (c) a nested-model comparison was performed to evaluate whether the full model fit the data significantly better than the nested model.

\section{Locus of Control (LOC)}

For Hypothesis 3, I predicted that cognitive load would moderate the association between LOC and data-visualization interpretation time, such that under conditions of higher cognitive load the association between LOC and interpretation time would be more positive. The full model that included the product terms did not fit the data significantly better than the nested model $(F 2,52=1.452, p=.243)$, and thus Hypothesis 3 was not supported. For Hypothesis 4, I predicted that cognitive load would moderate the association between LOC and datavisualization interpretation accuracy, such that under conditions of higher cognitive load the association between LOC and interpretation accuracy would be less positive. The addition of the product terms did not result in a significantly better fitting model $(F 2,52=3.133, p=.052)$, and thus Hypothesis 3 was not supported. 


\section{Neuroticism}

For Hypothesis 5, I predicted that cognitive load would moderate the association between neuroticism and data-visualization interpretation time, such that under conditions of higher cognitive load the association between neuroticism and interpretation time would be less negative. The full model that included the product terms did not fit the data significantly better than the nested model $(F 2,52=3.159, p=.051)$, and thus Hypothesis 5 was not supported. For Hypothesis 6, I predicted that cognitive load would moderate the association between neuroticism and data-visualization interpretation accuracy, such that under conditions of higher cognitive load the association between neuroticism and interpretation accuracy would be more negative. The full model that included the product terms did not fit the data significantly better than the nested model $(F 2,52=1.840, p=.169)$, and thus Hypothesis 6 was not supported.

\section{Extraversion}

For Hypothesis 7, I predicted that cognitive load would moderate the association between extraversion and data-visualization interpretation time, such that under conditions of higher cognitive load the association between extraversion and interpretation time would be less negative. The full model that included the product terms did not fit the data significantly better than the nested model $(F 2,52=.826, p=.444)$, and thus Hypothesis 7 was not supported. For Hypothesis 8, I predicted that cognitive load would moderate the association between extraversion and data-visualization interpretation accuracy, such that under conditions of higher cognitive load the association between extraversion and interpretation accuracy would be more negative. The full model that included the product terms did not fit the data significantly better than the nested model $(F=2,52=.410, p=.666)$, and thus Hypothesis 8 was not supported. 


\section{Openness to Experience}

For Hypothesis 9, I predicted that cognitive load would moderate the association between openness to experience and data-visualization interpretation time, such that under conditions of higher cognitive load the association between openness to experience and interpretation time would be less negative. The full model that included the product terms did not fit the data significantly better than the nested model $(F 2,52=.104, p=.901)$, and thus Hypothesis 9 was not supported. For Hypothesis 10, I predicted that cognitive load would moderate the association between openness to experience and data-visualization interpretation accuracy, such that under conditions of higher cognitive load the association between openness to experience and interpretation accuracy would be less positive. The full model that included the product terms did not fit the data significantly better than the nested model $(F 2,52=.295, p=.746)$, and thus Hypothesis 10 was not supported.

\section{Supplementary Analyses}

In supplementary analyses, I relaxed the time constraint applied to each question to 90 seconds instead of 60 seconds. This resulted in more observations being retained. The associated zero-order correlations and means and standard deviations appear in Table 3. The percentage of correct responses was as follows: $6.9 \%(n=4)$ answered 0 correctly, 29.3\% $(n=17)$ answered 1 correctly, 34.5\% $(n=20)$ answered 2 correctly, $24.1 \%(n=14)$ answered 3 correctly, and finally $5.2 \%(n=3)$ answered all 4 correctly. With the exception of Hypothesis 6 , all of the hypotheses remained unsupported. Hypothesis 6 received partial support when the time constraint was relaxed to 90 seconds. Specifically, cognitive load was found to moderate the association between neuroticism and data-visualization interpretation accuracy $(F 2,52=3.416, p=.040)$; however, the form of the interaction was not exactly as predicted. As shown in Table 4 and 
Figure 6, the association between neuroticism and interpretation accuracy was significant and negative for participants from the moderate cognitive load group $(S S=-.87, p<.05)$, but the significant slopes were not significant for the control group $(S S=.07, p=.82)$ or the high cognitive-load group $(S S=-.33, p=.21)$. The association found for those who experienced moderate cognitive load suggests that there was a negative association between neuroticism and interpretation accuracy, such that those that scored higher in the neuroticism scale interpreted information displayed in data visualizations with less accuracy. The amount of incremental variance explained by the product terms in relation to interpretation accuracy when controlling for the additive effects would generally be considered to be medium in magnitude $\left(\Delta R^{2}=.093\right)$.

As an exploratory interpretation measure and using the original 60 -second time constraint, I tested same models as above with the confidence-accuracy data-visualization interpretation measure. A one-way ANOVA was used to determine whether there were significant differences in the overall confidence levels between the three groups. The results showed confidence-accuracy levels did not differ between the three cognitive-load groups ( $F$ $2,55=2.18, p=.12$ ). Additionally, confidence measures were further analyzed by conducting a series of MMLRs using nested-model comparisons for each individual-difference variable to identify whether cognitive load moderated the associations between each individual-difference variable and confidence-accuracy. Cognitive load did not moderate the association between LOC and confidence-accuracy levels $(F 2,52=1.848, p=.168)$. Cognitive load did not moderate the association between neuroticism and confidence-accuracy levels $(F 2,52=2.932, p=.062)$. Cognitive load did not moderate the association between extraversion and confidence-accuracy levels $(F 2,52=.680, p=.511)$. Cognitive load did not moderate the association between openness to experience and confidence-accuracy levels $(F 2,52=.987, p=.379)$. 
Table 3

Means, standard deviations, and zero-order correlations with confidence intervals

\begin{tabular}{|c|c|c|c|c|c|c|c|c|}
\hline Variable & $M$ & $S D$ & 1 & 2 & 3 & 4 & 5 & 6 \\
\hline 1. Speed & 36.41 & 11.79 & & & & & & \\
\hline 2. Accuracy & 1.91 & 1.01 & $\begin{array}{l}.22 \\
{[-.04, .46]}\end{array}$ & & & & & \\
\hline 3. Locus of control & 3.79 & .70 & $\begin{array}{l}-.12 \\
{[-.37, .14]}\end{array}$ & $\begin{array}{l}-.01 \\
\quad[-.27, .25]\end{array}$ & & & & \\
\hline 4. Neuroticism & 2.98 & .81 & $\begin{array}{c}-.28^{*} \\
{[-.50,-.03]}\end{array}$ & $\begin{array}{c}-.37 * * \\
{[-.57,-.12]}\end{array}$ & $\begin{array}{c}-.04 \\
{[-.29, .22]}\end{array}$ & & & \\
\hline 5. Extraversion & 2.91 & .87 & $\begin{array}{l}-.08 \\
{[-.33, .18]}\end{array}$ & $\begin{array}{c}.09 \\
{[-.18, .34]}\end{array}$ & $\begin{array}{c}.01 \\
{[-.25, .27]}\end{array}$ & $\begin{array}{c}.05 \\
{[-.22, .30]}\end{array}$ & & \\
\hline 6. Openness to experience & 3.63 & .79 & $\begin{array}{c}.10 \\
{[-.16, .35]}\end{array}$ & $\begin{array}{l}-.00 \\
{[-.26, .26]}\end{array}$ & $\begin{array}{c}-.06 \\
{[-.31, .20]}\end{array}$ & $\begin{array}{l}-.05 \\
{[-.30, .21]}\end{array}$ & $\begin{array}{c}-.11 \\
{[-.36, .15]}\end{array}$ & \\
\hline 7. Confidence & 2.87 & 40.24 & $\begin{array}{l}.22 \\
{[-.05, .45]}\end{array}$ & $\begin{array}{c}.88 * * \\
{[.81, .93]}\end{array}$ & $\begin{array}{c}-.03 \\
{[-.28, .23]}\end{array}$ & $\begin{array}{c}-.33^{*} \\
{[-.54,-.07]}\end{array}$ & $\begin{array}{c}.09 \\
{[-.18, .34]}\end{array}$ & $\begin{array}{c}.05 \\
{[-.21, .30]}\end{array}$ \\
\hline
\end{tabular}

Note. $n=58 . M$ and $S D$ are used to represent mean and standard deviation, respectively. Values in square brackets indicate the $95 \%$ confidence interval for each correlation. The confidence interval is a plausible range of population correlations that could have caused the sample correlation (Cumming, 2014). * indicates $p<.05$. ** indicates $p<.01$.

Table 4

Nested-model comparison with interpretation accuracy as the outcome 


\begin{tabular}{|c|c|c|c|c|c|c|}
\hline Predictor & $b$ & $\begin{array}{c}b \\
95 \% \mathrm{CI} \\
{[\mathrm{LL}, \mathrm{UL}]}\end{array}$ & $s r^{2}$ & $\begin{array}{c}s r^{2} \\
95 \% \mathrm{CI} \\
{[\mathrm{LL}, \mathrm{UL}]}\end{array}$ & Fit & Difference \\
\hline Intercept & $3.62 * *$ & {$[2.60,4.63]$} & & & & \\
\hline $\begin{array}{l}\text { Dummy Variable (High } \\
\text { Cognitive Load) }\end{array}$ & $-.61 *$ & {$[-1.21,-.01]$} & .06 & {$[-.05, .17]$} & & \\
\hline $\begin{array}{l}\text { Dummy Variable (Moderate } \\
\text { Cognitive Load) }\end{array}$ & -.43 & {$[-1.04, .19]$} & .03 & {$[-.05, .11]$} & & \\
\hline Neuroticism & $-.45^{* *}$ & {$[-.76,-.14]$} & .13 & {$[-.03, .28]$} & $\begin{array}{l}R^{2}=.200 * * \\
95 \% \mathrm{CI}[.02, .34]\end{array}$ & \\
\hline Intercept & $2.08 *$ & {$[.33,3.84]$} & & & & \\
\hline $\begin{array}{l}\text { Dummy Variable (High } \\
\text { Cognitive Load) }\end{array}$ & .55 & {$[-1.86,2.97]$} & .00 & {$[-.02, .03]$} & & \\
\hline $\begin{array}{l}\text { Dummy Variable (Moderate } \\
\text { Cognitive Load) }\end{array}$ & $2.30 *$ & {$[.07,4.53]$} & .06 & {$[-.04, .16]$} & & \\
\hline Neuroticism & .07 & {$[-.51, .64]$} & .00 & {$[-.01, .01]$} & & \\
\hline $\begin{array}{l}\text { Cognitive Load) X } \\
\text { Neuroticism }\end{array}$ & -.40 & {$[-1.17, .38]$} & .01 & {$[-.04, .07]$} & & \\
\hline $\begin{array}{l}\text { Dummy Variable (Moderate } \\
\text { Cognitive Load) X } \\
\text { Neuroticism }\end{array}$ & $-.93 *$ & {$[-1.66,-.20]$} & .09 & {$[-.04, .21]$} & & \\
\hline & & & & & $\begin{array}{l}R^{2}=.293 * * \\
95 \% \text { CI }[.05, .42]\end{array}$ & $\begin{array}{l}\Delta R^{2}=.093 * \\
95 \% \mathrm{CI}[-.03, .22]\end{array}$ \\
\hline
\end{tabular}

Note. $n=58$. A significant $b$-weight indicates the semi-partial correlation is also significant. $b$ represents unstandardized regression weights. $s r^{2}$ represents the semi-partial correlation squared. $L L$ and $U L$ indicate the lower and upper limits of a confidence interval, respectively. $*$ indicates $\mathrm{p}<.05 . * *$ indicates $\mathrm{p}<.01$. 


\section{Figure 6}

Simple slopes plot of significant interaction between neuroticism and cognitive load in relation to data-visualization interpretation accuracy

4

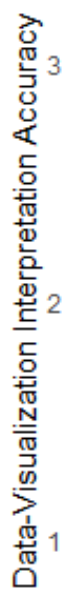

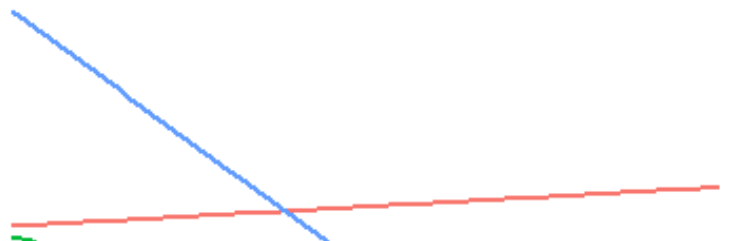

Cognitive Load

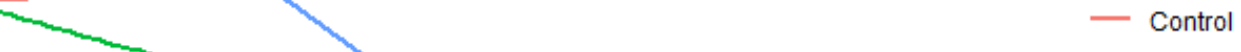

- Moderate

- High

0

1

2

Neuroticism

4

5 


\section{Discussion}

All the hypotheses that were tested were not supported, except for Hypothesis 6 from the supplementary analysis, which could be due to low levels of internal consistency reliability and/or a lower-than desired statistical power due to a relatively small sample $(n=58)$. There may be other underlying user characteristics that affected this sample which could better explain the null results. The significant moderation found within the supplementary analyses indicates that when cognitive load was moderate, those with highest neuroticism tended to interpret data visualizations with the lowest accuracy. This is partially aligned with the proposed hypothesis, but unexpectedly there was no association between neuroticism and interpretation accuracy for those who experienced a high level of cognitive load. Interestingly, evidence of this moderation was only found when the time constraint was relaxed from 60 to 90 seconds.

\section{Implications}

From a theoretical perspective, researchers interested in data visualizations should consider the impact that a distracting environment has on participants who have higher levels of neuroticism. These distracting environments contribute to extraneous cognitive load and may affect interpretation accuracy. Another consideration is the effect that task switching can have on these individuals. If participants are supposed to remember certain minutiae of an old task while they go on to complete another task, then this may further affect interpretation accuracy since they would have less working memory resources available. This relationship was only found within the moderate level cognitive load group, which may be indicative of either a small sample, or a faulty manipulation for the high-level cognitive load group.

From a practical perspective, developers of these data visualizations should consider how cognitive load stemming from the work environment and HR managers' level of neuroticism 
might interaction and affect interpretation accuracy. Given that cognitive load stems from the idea that humans have a limited working memory capacity, it would make sense to try and limit the amount of working-memory demands HR managers experience when they are tasked with interpreting a data visualization - especially for those who have higher levels of neuroticism. This could be as simple as making sure that the users are not working on multiple tasks at once or not working in a distracting workspace. This could allow for more working memory demands to be directed at the task at hand, which could lead to better interpretation time and effectiveness.

\section{Limitations}

There were several limitations in this study that should be kept in mind when interpreting the results. First, the method of simulating cognitive load by means of a working-memory task may not fully encapsulate all the working memory demands that individuals experience in the workplace, which may affect the generalizability of these results. Surely the intricacies of the workplace go further than simply memorizing an alphanumeric sequence. Second, only two datavisualization display types were used: bar graph and line graph. These are two of the most common data visualization display types, but using more complex data-visualization display types - such as heatmaps or alluvial diagrams - could broaden the implications of the present study. Third, this sample included undergraduate business students as opposed to HR managers. The business students likely had varying levels of exposure to these types of data visualizations and to manage principles in general, whereas HR managers likely have strong domain and context knowledge to facilitate their interpretations. Fourth, the relatively small sample size may have resulted in lower-than-desired statistical power and thus my ability to find true effects was reduced. Finally, internal consistency reliabilities for some of the personality measures were less than the conventional .70 cutoff, which may have limited the magnitude of the effects I observed. 


\section{Future Directions}

Future researchers may be interested in experimenting with different data visualization display types to see how users analyze them in a similar type of study. With the massive advances in computational power, there is far more that we can accomplish with data visualizations beyond the use of static images. Researchers may want to explore how users interact with data visualizations that can be easily manipulated by the user by using dashboards. This could generate insight on how different users explore certain data visualizations depending on their unique user characteristics.

To expand upon this present study, future researchers may look at incorporating different types of audio or visual forms of cognitive load that better simulate the workplace, such as an email notification that comes up in the middle of the task. Additionally, it may be interesting to add different types of questions, as all the vignettes in this study contained single choice response questions. By introducing different types of questions, such as a rank order with weighted responses or short answer response, there may be more room for interpretability. This could create more insights into how users analyze data visualizations, and how this affects their overall interpretation efficiency and effectiveness.

\section{Conclusion}

This thesis explored whether cognitive load impacts data visualization effectiveness and efficiency, as well as investigated whether cognitive load moderated the association between individual differences and interpretation time and accuracy. A pilot study was conducted to test these claims, and although all the tested hypotheses were non-significant, there are still significant contributions to consider. This work adds to the research around CLT by applying these theoretical principles to an HRM context. This thesis has also contributed to the growing 
body of literature around individual differences and data visualizations. Finally, this work has received partial support in the discovery that cognitive load moderates the association between neuroticism and interpretation accuracy. This interaction effect was only present within one of the groups, and it was only found after supplementary analyses, nonetheless the interaction effect found was significant. This finding indicates the need for future research into this area to further explore this interaction.

Overall, the contributions within this thesis help us move toward a better understanding of how cognitive load and individual differences interact when working with data visualizations. Data visualizations can be incredibly powerful decision-support tools; however, it is imperative that the intended message is not missed. If HR managers within an organization have higher levels of neuroticism and they are experiencing moderate levels of cognitive load, then they may have poorer interpretation accuracy. To mitigate the likelihood of this happening, managers should be mindful of their workplace environment when they are analyzing data visualizations. Extraneous workplace cognitive demands can take away from interpreting data visualizations and by eliminating them you could allow for more working memory resources to be allocated towards the primary task at hand.

For those interested, there is a direct link that includes all the $\mathrm{R}$ code used in this thesis within the appendix. The code contains each step that was mentioned along with annotations explaining what I did. 


\section{References}

Bailey, J., \& Pregill, L. (2014). The history and practice of information visualization. Journal of the Art Libraries of North America, 33(2), 168-191.

Barrick, M. R., \& Mount, M. K. (1991). The Big Five personality dimensions and job performance: A meta-analysis. Personnel Psychology, 44(1), 1-26. https://doi.org/10.1111/j.1744-6570.1991.tb00688.x

Brown, E. T., Ottley, A., Zhao, H., Lin, Q., Souvenir, R., Endert, A., \& Chang, R. (2014). Finding Waldo: Learning about users from their interactions. IEEE Transactions on Visualization and Computer Graphics, 20(12), 1663-1672. https://doi.org/10.1109/TVCG.2014.2346575

Caughlin, D. E., \& Bauer, T. N. (2019). Data visualizations and human resource management: The state of science and practice. In M. R. Buckley, A. R. Wheeler, J. E. Baur, \& J. R. B. Halbesleben (Eds.), Research in personnel and human resources management (pp. 89131, Vol. 37). West Yorkshire, UK: Emerald Publishing Limited.

Cumming, G. (2014). The new statistics: Why and how. Psychological Science, 25(1), 729. https://doi.org/10.1177/0956797613504966

DDI, The Conference Board, \& EY. (2018). Global Leadership Forecast 2018: 25 research insights to fuel your people strategy. Pittsburgh, PA: DDI.

Donnellan, M. B., Oswald, F. L., Baird, B. M., \& Lucas, R. E. (2006). The mini-IPIP scales: Tiny-yet-effective measures of the Big Five factors of personality. Psychological Assessment, 18(2), 192-203. http://dx.doi.org.proxy.lib.pdx.edu/10.1037/10403590.18.2.192 
Goldberg, L. R. (1992). The development of markers for the Big-Five factor structure. Psychological Assessment, 4, 26-42.

Goldberg, L. R. (1999). A broad-bandwidth, public domain, personality inventory measuring the lower-level facets of several five-factor models. In I. Mervielde, I. Deary, F. De Fruyt, \& F. Ostendorf (Eds.), Personality Psychology in Europe, Vol. 7 (pp. 7-28). Tilburg, The Netherlands: Tilburg University Press.

Green, T. M., \& Fisher, B. (2010). Towards the personal equation of interaction: The impact of personality factors on visual analytics interface interaction. 2010 IEEE Symposium on Visual Analytics Science and Technology, 203-210. https://doi.org/10.1109/VAST.2010.5653587

Green, T. M., \& Fisher, B. (2012). Impact of personality factors on interface interaction and the development of user profiles: Next steps in the personal equation of interaction. Information Visualization, 11(3), 205-221.

Lavie, N. (2010). Attention, distraction, and cognitive control under load. Current Directions in Psychological Science, 19(3), 143-148.

Liu, Z., Crouser, R. J., \& Ottley, A. (2020). Survey on individual differences in visualization. Computer Graphics Forum, 39(3), 693-712. https://doi.org/10.1111/cgf.14033

Murphy, G., Groeger, J. A., \& Greene, C. M. (2016). Twenty years of load theory-Where are we now, and where should we go next? Psychonomic Bulletin \& Review, 23(5), 13161340. https://doi.org/10.3758/s13423-015-0982-5

Myszkowski, N., Storme, M., Davila, A., \& Lubart, T. (2015). Managerial creative problem solving and the Big Five personality traits: Distinguishing divergent and convergent 
abilities. Journal of Management Development, 34(6), 674-684.

https://doi.org/10.1108/JMD-12-2013-0160

Oh, I.-S., Wang, G., \& Mount, M. K. (2011). Validity of observer ratings of the five-factor model of personality traits: A meta-analysis. Journal of Applied Psychology, 96(4), 762773.

Paas, F. G. (1992). Training strategies for attaining transfer of problem-solving skill in statistics: A cognitive-load approach. Journal of Educational Psychology, 84(4), 429-434. http://dx.doi.org.proxy.lib.pdx.edu/10.1037/0022-0663.84.4.429

Pervin, L. A., Cervone, D., \& John, O. P. (2005). Personality: Theory and Research (9th ed.). Hoboken, NJ: John Wiley \& Sons.

Reb, J., \& Cropanzano, R. (2007). Evaluating dynamic performance: The influence of salient Gestalt characteristics on performance ratings. Journal of Applied Psychology, 92(2), 490-499. https://doi.org/10.1037/0021-9010.92.2.490

Roels, R., Baeten, Y., \& Signer, B. (2017). Interactive and narrative data visualisation for presentation-based knowledge transfer. In G. Costagliola, J. Uhomoibhi, S. Zvacek, \& B. M. McLaren (Eds.), Computers Supported Education (Vol. 739, pp. 237-258). Springer International Publishing. https://doi.org/10.1007/978-3-319-63184-4_13

Rotundo, M., \& Sackett, P. R. (2002). The relative importance of task, citizenship, and counterproductive performance to global ratings of job performance: A policy-capturing approach. Journal of Applied Psychology, 87(1), 66-80. https://doi.org/10.1037/00219010.87 .1 .66 
Sinar, E. F. (2015). Data visualization. In S. Tonidandel, E. B. King, \& J. M. Cortina (Eds.), Big data at work: The data science revolution and organizational psychology (pp. 115-157). New York, NY: Routledge.

Spector, P. E. (1982). Behavior in organizations as a function of employee locus of control. Psychological Bulletin, 91, 482-497. https://doi.org/10.1037/0033-2909.91.3.482

Spector, P. E. (1988). Development of the Work Locus of Control Scale. Journal of Occupational Psychology, 61(4), 335-340. doi:10.1111/j.2044-8325.1988.tb00470.x

Stanley, D. (2021). apaTables: Create American Psychological Association (APA) style tables. R package version 2.0.8. https://CRAN.R-project.org/package=apaTables

Sweller, J. (1994). Cognitive load theory, learning difficulty, and instructional design. Learning and Instruction, 4(4), 295-312. https://doi.org/10.1016/0959-4752(94)90003-5

Sweller, J. (2010). Cognitive load theory: Recent theoretical advances. In J. L. Plass, R. Moreno, \& R. Brunken (Eds.), Cognitive Load Theory (pp. 29-47). Cambridge University Press. https://doi.org/10.1017/CBO9780511844744.004

Tufte, E. R. (1983). The visual display of quantitative information. Cheshire, CT: Graphics Press van Merriënboer, J. J. G., \& Sweller, J. (2005). Cognitive load theory and complex learning: Recent developments and future directions. Educational Psychology Review, 17(2), 147177. https://doi.org/10.1007/s10648-005-3951-0

Weiss, H., \& Sherman, J. (1973). Internal-external control as a predictor of task effort and satisfaction subsequent to failure. Journal of Applied Psychology, 57(2), 132-136. http://dx.doi.org.proxy.lib.pdx.edu/10.1037/h0037054

Ziemkiewicz, C., \& Kosara, R. (2009). Preconceptions and individual differences in understanding visual metaphors. Computer Graphics Forum, 28(3), 911-918. 
https://doi.org/10.1111/j.1467-8659.2009.01442.x 


\section{Appendix}

\section{Annotated R Code}

All the R code used in this thesis has been uploaded to the Open Science Framework (OSF.io) and it is publicly available. Here is a direct link that leads you to the project where the code is stored: https://osf.io/muejf/?view_only=1e729948a8b54ec99d2ddda82859d51d. 